\title{
Performance of COFDM Using Turbo Codes
}

\author{
Lamine Sylla, Paul Fortier, Huu Tuê Huynh \\ Department of Electrical and Computer Engineering \\ Laval University, Ste-Foy, Quebec Canada G1K 7P4 \\ (418) 656-3555, (418) 656-3159 fax \\ e-mail: fortier@gel.ulaval.ca
}

The main presenter of this paper will be Paul Fortier. Should the paper be accepted, Dr. Fortier will attend the conference.

AbSTRACT - In this paper, we discuss and present simulation results for the performances of COFDM systems using turbo codes and operating in a mobile radio environment. Results show that the echo power has a much stronger influence than the guard time interval or echo delay. This scheme is shown to be an excellent solution for transmission over bandlimited mobile channels.

Primary Technical Subject Area: Radio Communication

Submitted to ICC-99 


\section{Introduction}

With the recent development of Personal Communication Systems (PCS), there is a need for bandwidth efficient schemes that perform well in additive noise as well as being robust in a fading environment. Turbo codes have been known to attain near Shannon limit performances in AWGN [8]. Coded Orthogonal Frequency Division Multiplexing (COFDM), originally developed for digital audio broadcasting, has been shown to be a very good scheme to combat fading and nonGaussian noise [9]. It is quite natural then to look at the combination of COFDM with turbo codes as a system architecture for mobile radio or indoor environments.

Since the first publications regarding turbo codes, various studies have been carried out. Most of these studies illustrated performances or dealt with the optimization of the structure of turbo codes using binary modulation. The requirement for higher information transmission rates on bandlimited channels have led some researchers to consider turbo codes with spectrally efficient modulations [1].

Turbo codes can also be used with trellis coded modulation (TCM) to increase the spectral efficiency [2]. The encoder uses two recursive systematic convolutional coders (RSC) of rate $R=l^{\prime}(l+1), l$ being even, and two interleavers for a throughput of $l$ bits $/ \mathrm{sec} / \mathrm{Hz}$ with $2^{2 l+2}$ QAM. The decoding is performed iteratively by the symbol MAP decoder followed by a bit reliability calculation module. Sometimes, these two RSCs are replaced by two Ungerboeck codes in their recursive form as component codes, and PSK takes the place of QAM [3]. This turbo TCM (TTCM) uses an interleaver operating over symbols of 2 bits for an 8 PSK modulation. The variables passed between symbol MAP decoders are vectors of four log-likelihood ratios (LLRs), one for each possible information group value.

This paper presents some results obtained with turbo codes and QAM modulation to be used with COFDM in the Rummler channel by applying the so called "pragmatic" approach [1].

The paper is organized as follows: Section 2 deals with the overall structure of the transmission system, including COFDM, turbo code and channel model. Section 3 presents and discusses the simulation results. The last section is devoted to some concluding remarks. 


\section{Overall structure of the transmission system}

The general architecture of the system is shown on Figure 1. In this scheme, turbo codes are used for channel encoding and COFDM is the modulation method.

\subsection{Turbo codes}

The encoder is made up of two RSCs with constraint length $k=3$ and polynomial generators $(5,7)$. The RSCs are separated by a uniform block interleaver. For short frame transmission applications, the interleaver is often set to a size of 192 bits which is compatible with the IS-95 CDMA cellular standard. In our case, a block size of 1022 bits has been used. This size, after adding 2 bits for terminating the RSCs, match the number of sub-carriers of COFDM, which is a power of 2. The output of the $1 / 3$ turbo encoder is punctured to obtain a $1 / 2$ coding rate.

The system presented here is different from the TCM approach in the sense that it uses a coding rate $R=1 / 2$ for a 16 point constellation. The output sequence of the encoder $\left(u_{1}, c_{1}, u_{2}, c_{2}, \ldots.\right)$ is modulated by two 4-ASK in quadrature resulting in a 16-QAM system where the $I$ and $Q$ components are independently coded (using Gray code). The ASK symbols $\left\{A_{k}, B_{k}\right\}$ are then transmitted through the channel.

The decoding of this multilevel modulation system is much simplified by use of the "pragmatic" approach [1]. The turbo decoder, implemented with the SOVA [4] algorithm, and optimized for binary modulation, can then be used. With this approach, the decoder must be preceded by a module that computes the LLR of each bit contained in an ASK symbol. The LLR can be expressed as follows:

$$
\Lambda\left(u_{k, i}\right)=\frac{\sigma^{2}}{2} \cdot \log \left(\frac{\sum_{i=1}^{2} \exp -\left(\frac{1}{2 \sigma^{2}} \cdot\left(X_{k}-a_{0, i}\right)^{2}\right)}{\sum_{i=1}^{2} \exp -\left(\frac{1}{2 \sigma^{2}} \cdot\left(X_{k}-a_{1, i}\right)^{2}\right)}\right)
$$

where $u_{k, 1}=u_{k}$ and $u_{k, 2}=c_{k}$ and $\left(a_{1, i}, a_{0, i}\right)$ are the realizations of symbol $A_{k}$ conditional to $u_{k, i}=1$ and $u_{k, i}=0$ respectively. A good approximation of this expression is given by 


$$
\begin{aligned}
& \Lambda\left(c_{k}\right)=\left|X_{k}\right|-2 \\
& \Lambda\left(u_{k}\right)=X_{k}
\end{aligned}
$$

Although this approximation can lead to good performances, the expression of $\Lambda\left(u_{k}\right)$ in (5) is an overestimation of its real value. A better approximation can be obtained by defining the bitmapping in one dimension of the constellation with unit energy as follows

$$
\begin{aligned}
A_{k} & =2 d \cdot\left(1-2 u_{k, 1}\right) \\
& +d \cdot\left(1-2 u_{k, 2}\right) \cdot \operatorname{sign}\left(1-2 u_{k, 1}\right)
\end{aligned}
$$

where $2 d$ is the distance between two points. This equation can be decomposed into two terms.

$$
\begin{aligned}
\Lambda\left(u_{k, 1}\right) & =2 d X_{k} \\
& +\frac{\sigma^{2}}{2} \cdot \log \left(\frac{\cosh \left(\frac{1}{2 \sigma^{2}} \cdot\left(4 d^{2}-2 d X_{k}\right)\right)}{\cosh \left(\frac{1}{2 \sigma^{2}} \cdot\left(4 d^{2}+2 d X_{k}\right)\right)}\right)
\end{aligned}
$$

$\Lambda\left(u_{k}\right)$ can now be approximated by the first term on the second part of this equation. Figure 1 show three curves of $\Lambda\left(u_{k}\right)$ versus $X_{k}$. The solid line represents the true expression in (1), the "**" line represents the first approximation in (2) and the "o" line the last approximation. The approximation in (4) is closer to its real value; as a consequence, it can be shown to give a better performance.

\subsection{COFDM}

Initially proposed more than 40 years ago and finally systematized by Weinstein [10], multicarrier modulation is a parallel transmission scheme. The bit stream to be transmitted is serially separated into $N_{\mathrm{c}}$ groups, each of which is used to modulate one of $N_{\mathrm{c}}$ sub-carriers. If the symbol rate is identical for every carrier, then all groups have the same length. The $N_{\mathrm{c}}$ modulated signals are added up to form one single modulated signal to be sent on the channel. When these sub-carriers are orthogonal over the signalling interval, these scheme is commonly named OFDM (orthogonal frequency division multiplexing). This OFDM, combined with some channel coding scheme 
is called COFDM. Figure 2 shows the general structure of a COFDM system.

In this paper, we are interested in using $M$-QAM to modulate each sub-carrier. When the number of sub-carriers is relatively high, this scheme becomes too complex to be implemented with common microelectronics components. On the other hand, it can be shown that the overall operation of the modem is equivalent to using FFT processing to obtain a composite signal, which in turn modulates a single carrier. The receiver uses, in the baseband equivalent mode, the filtering and sampling actions to deliver the demodulated signals. Therefore, in practice, COFDM modems use this FFT technique to reduce the hardware complexity. The output of the IFFT processor, corresponding to a COFDM symbol, is serially transmitted. To avoid the influence of echoes due to multipath fading, the transmitter adds a guard time interval after the COFDM symbol, during which no information symbol is transmitted. Normally, the guard time interval must be larger than the longest delay of the multipath.

\subsection{Channel model}

Generally, for purpose of simulation, two approaches are used to model a mobile radio channel. The first one deals with the multipath phenomenon and the second one deals, in addition to multipath, with frequency selectivity [7]. A Rayleigh flat fading channel can be used to simulate the multipath phenomenon. In this case, the received symbol, $\left(x_{k}, y_{k}\right)$, is given by

$$
\begin{aligned}
& x_{k}=\alpha_{k} \cdot A_{k}+i_{k} \\
& y_{k}=\beta_{k} \cdot B_{k}+q_{k}
\end{aligned}
$$

where $\left(\alpha_{k}, \beta_{k}\right)$ are two independent Rayleigh distributed random variables and $\left(i_{k}, q_{k}\right)$ are the additive white Gaussian noise components with variance $\sigma^{2}$.

The frequency selectivity can be introduced by means of intersymbol interference (ISI). In an air-to-ground communication, the channel is characterized by a direct line of sight (LOS) and a multipath component with a relative delay [5]. This delayed path creates some ISI on the received signal

$$
\begin{aligned}
& x_{k}=A_{k}+\alpha_{k-n} \cdot A_{k-n}+i_{k} \\
& y_{k}=B_{k}+\beta_{k-n} \cdot B_{k-n}+q_{k}
\end{aligned}
$$


with $n$ representing the relative delay of the diffuse component. This model fits the Rician fading channel.

For applications in microwave LOS radio channels, a similar model has been considered, the Rummler channel which is a two path model [6]

$$
H(f)=a\left(1-b \cdot e^{-j 2 \pi\left(f-f_{0}\right) \tau_{0}}\right)
$$

where $a$ is the overall attenuation, and the term within parenthesis represents the interference between two rays having a relative delay $\tau_{0}$ and producing a minimum amplitude at the notch frequency $f_{0} . b$ is the relative amplitude of the multipath ray.

With high speed transmission, the mobile radio channel can be considered as quasi-stationary; this means that for a short period of time, the channel's physical parameters do not change. The channel impulse response corresponds to a sum of echoes with random arrival times and random amplitudes with a given power profile. Over the period of stationarity, these parameters can be considered fixed. In such a situation, the channel can be considered as a fixed multipath fading one. With this hypothesis, we can use Rummler's arguments to show that a two ray channel model fits quite well to the physical situation [6].

The outputs of the channel are coherently demodulated and the soft inputs of the decoder are then $\left(X_{k}, Y_{k}\right)$.

\section{Simulations results and discussion}

This work is the first step of a more important project. Given the fact that interleaving is already exploited by turbo codes, no attention is paid to time or frequency interleavers as normally used in COFDM schemes. Furthermore, no equalization or any channel correction are performed. The simulations have been carried out using the Signal Processing WorkSystem (SPW) package. The BER was computed after 3 iterations of the decoder. The number of simulations is defined to insure that the precision of the BER estimation is less than $1 \%$ with a confidence interval less than 5\%. The system parameters are chosen for a particular real situation where some transmission speed and bandwidth must be respected. As previously discussed, the receiver uses the approximation given by (4) for the LLRs in the decoding procedure.

In the following, the influence of the delay, $\tau_{0}$, the guard time interval, $T_{g}$, and the echo rela- 
tive amplitude, $b$, are successively discussed.

Figure 3 shows the BER performances of 16-QAM COFDM with a fixed relative guard time interval $\left(T_{g} / \tau_{0}=4 / 3\right)$ for different delays and different echo amplitudes. We observe that for a sufficient guard time interval, the echo delay does not sensibly affect the performance of the system. This result seems quite natural, due to the fact that the guard time interval makes the echoes die out before the next symbol. The intersymbol interference due to multipath is thus minimized. On the other hand, the echo relative amplitude seriously influences the behavior of the system. The echo, given a sufficient guard time, does not affect the next symbol; however, it directly affects the current symbol. Its influence is clearly observed on Figure 3. For of BER equal to $10^{-4}$, there is a loss of $3 \mathrm{~dB}$ in $E_{b} / N_{o}$ when $b$ increases from 0.25 to 0.4 . In fact, as the system under study does not include any channel equalization, the influence of the echo cannot be eliminated.

The influence of the guard time interval on the performance is shown in Figure 4. This figure presents, for various values of $T_{g} / \tau_{0}$ with fixed echo delay and echo relative amplitude, the BER curves. The worst case corresponds to the absence of a guard time interval. When a guard time interval is introduced, the performance is clearly improved. It is observed that when the guard time interval is larger than the echo delay, the performance quickly reaches its limit. It can be safely said that there is very little gain in SNR when $T_{g} / \tau_{0}$ goes beyond 1.1 .

\section{Conclusion}

This paper presented some preliminary results on the behavior of COFDM using turbo codes. It was shown that with a simple architecture the system offers quite acceptable performances. To improve the observed performances, more sophisticated actions should be taken into account. There are two interesting elements that can be considered. First, we can examine if the performances are enhanced by the introduction of a Reed-Solomon inner code. As seen previously, the echo power has a very strong influence on the overall system. To improve the BER performance we must find an effective way to minimize its effect. We can thus consider an equalizer, which should be chosen in such a way that the hardware complexity would not increase too much. One of simple ways to implement this is to introduce amplitude and phase compensation for each subcarrier. Another problem, although difficult, but very interesting to consider, is the design of good turbo codes for mobile channels. Finally, more sophisticated mobile radio channels should be considered. 


\section{References}

[1] S. Goff, A. Glavieux, and C. Berrou, "Turbo-codes and high spectral efficiency modulation," Proc, IEEE Int. Conf. on Commun., pp. 645-9, May 1994.

[2] S. Benedetto, D. Divsalar, G. Montorsi, and F. Pollara, "Parallel concatenated trellis coded modulation,” Proc, IEEE Int. Conf. on Commun., pp. 974-8, May 1996.

[3] P. Robertson and T. Worz, "Coded modulation scheme employing turbo codes," Electronics Letters, vol. 31, pp.1546-7, Aug. 31st, 1995.

[4] J. Hagenauer and L. Papke, "Decoding turbo codes with the soft-output Viterbi algorithm (SOVA),” Proc, IEEE Int. Symp. on Inform. Theory, p. 164, June 1994.

[5] J. Proakis, "Digital Communications", third edition. New York: McGraw-Hill, Inc.,1995.

[6] W. D. Rummler et al., "Multipath fading channels models for microwave digital radio", IEEE Communication Magazine, vol. 24, Nov. 1996.

[7] W. C. Lee, “Mobile Communication Engineering”, McGraw-Hill, 1982.

[8] C. Berrou, A. Glavieux and P. Thitimajshima, "Near Shannon limit error-correcting coding and decoding: Turbo-codes", Proc. IEEE ICC-93, pp. 1064-1070.

[9] H. T. Huynh, P. Fortier, G.-Y. Delisle, "Influence of a Class of Man-Made Noise on QAM Multicarrier Systems", Seventh Communication Theory Mini-Conference (Globecom'98), Sydney, Australia, 8-12 November, 1998.

[10] S. B. Weinstein, "Data Transmission by Frequency-Division Multiplexing Using the Discrete Fourier Transform”, IEEE Trans. Commun. vol. COM-19, no. 5, pp. 628-3, 1971. 


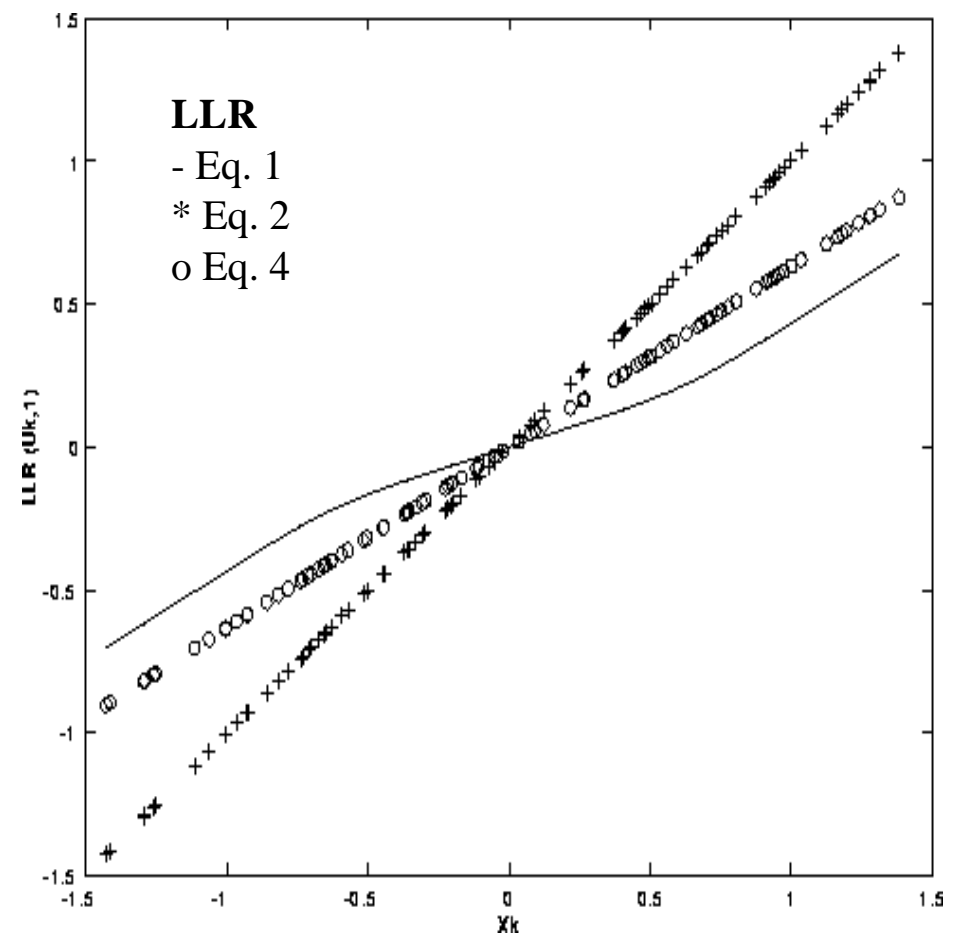

Figure 1: $\Lambda\left(u_{k}\right)$ versus $X_{k}$.



Figure 2: General structure of a COFDM system. 


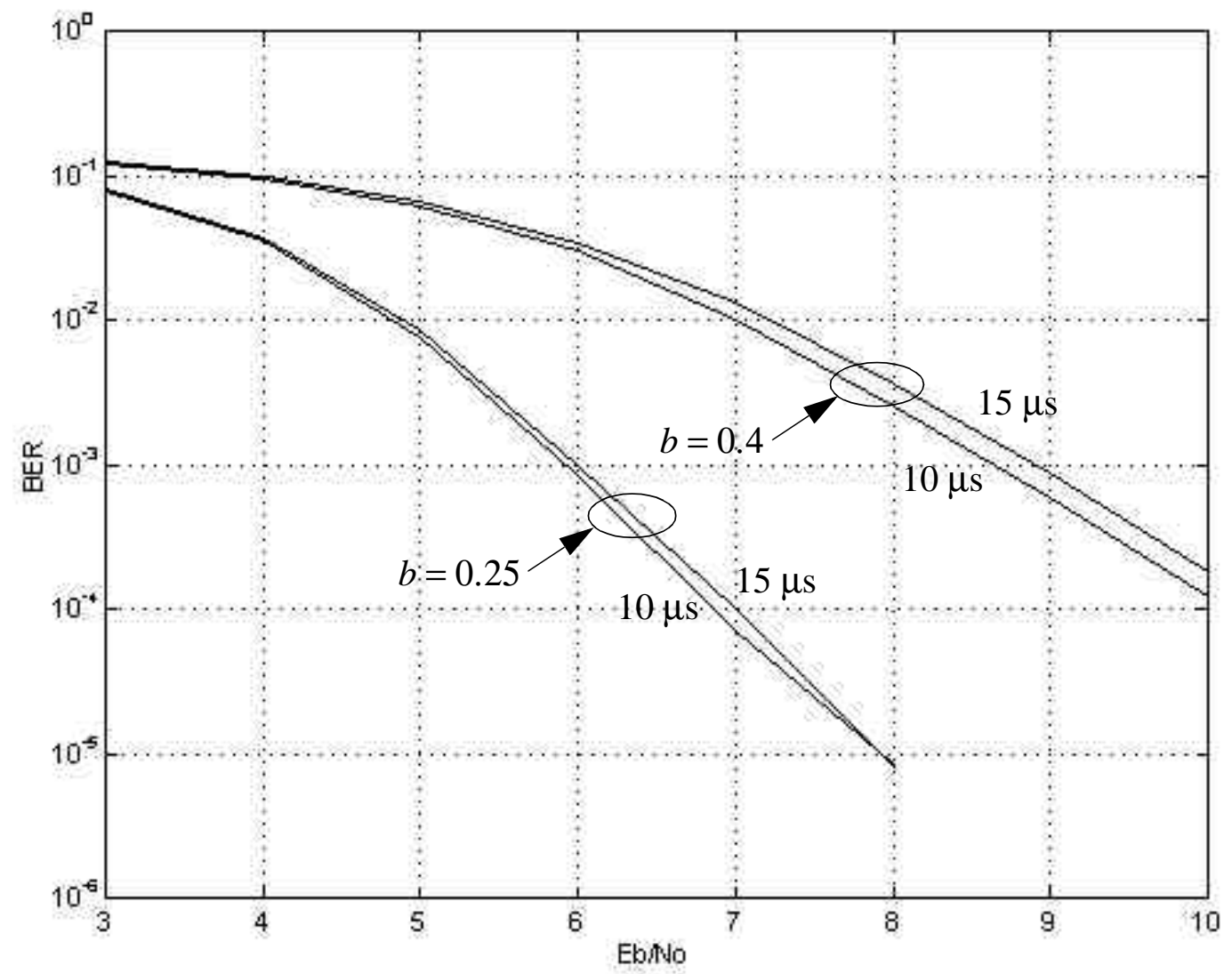

Figure 3: BER performances of 16-QAM COFDM for $T_{g} / \tau_{0}=4 / 3$. 


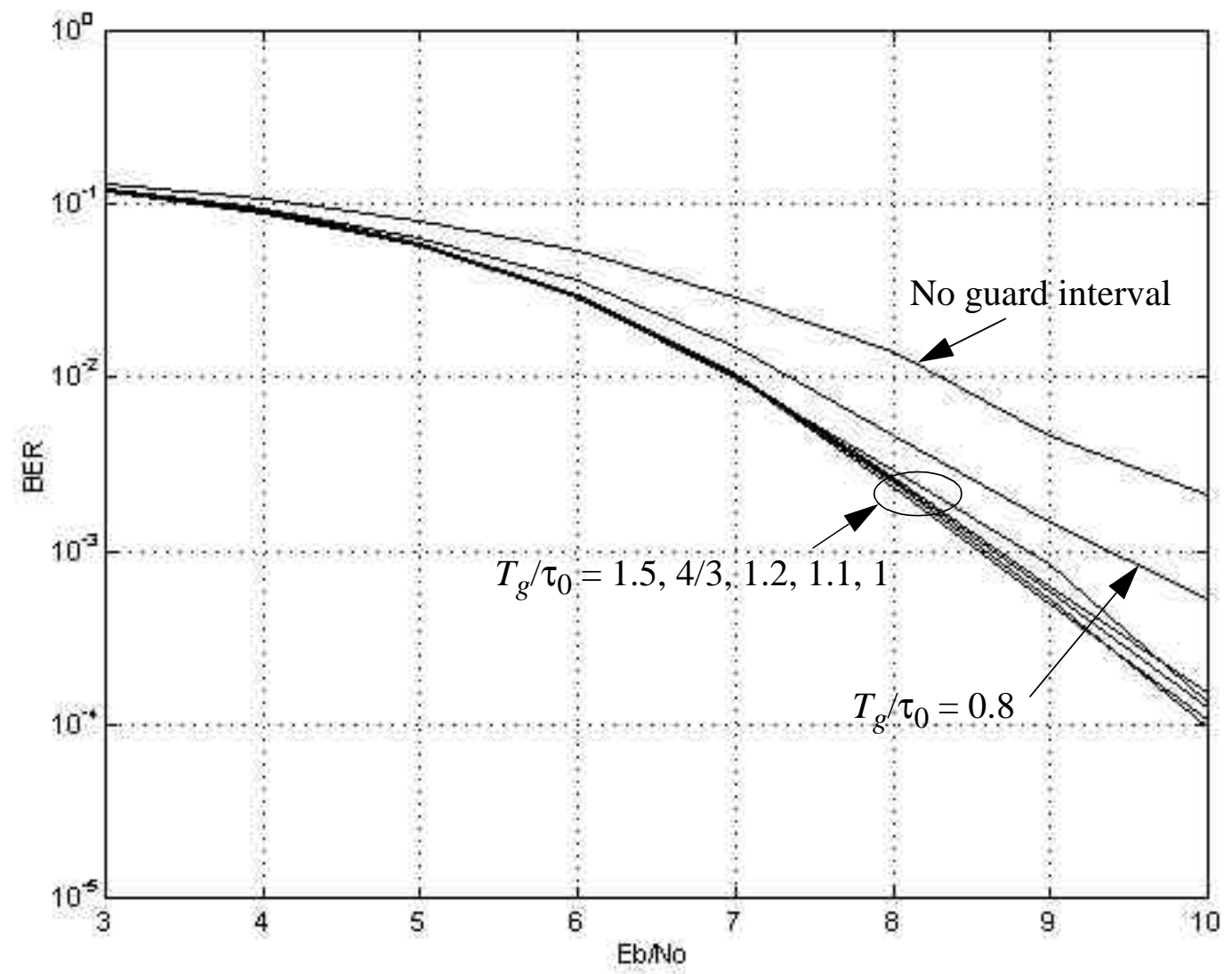

Figure 4: BER performances of 16-QAM COFDM for $\tau_{0}=15 \mu \mathrm{s}, b=0.4$, and various values of $T_{g} / \tau_{0}$. 